\title{
LETTER OPEN Hepatic microRNA-126 deficiency restrains liver regeneration through p53 pathway in mice
}

\author{
Signal Transduction and Targeted Therapy (2021)6:32
}

\section{Dear Editor,}

Emerging evidences show close associations between miRNAs and liver regeneration. MiR-126 is known as a governor of vascular integrity and angiogenesis. MiR-126 is involved in the self-renewal of hematopoietic stem cells (HSCs) and leukemia stem cells. ${ }^{1,2}$ MiR-126 also plays a vital role in hepatocellular carcinogenesis, ${ }^{3}$ however, the functions of miR-126 in liver regeneration were still unclear.

To explore hepatic functions of miR-126, we developed miR-126 liver specific knockout (LKO) mice (Supplementary Fig. 1a). MiR126 was almost undetectable in the hepatocytes of miR-126 LKO mice (Supplementary Fig. 1b, c, Supplementary Fig. 2a). The body weights showed no differences between two groups (Supplementary Fig. 2b). The liver to body weights and hepatic morphologies were also comparable between two groups (Supplementary Fig. 2c, d). The liver injury related index, alanine aminotransferase (ALT), aspartate aminotransferase (AST), albumin (ALB), total protein (TP), and total bile acids (TBA) levels in miR-126 LKO mice were all comparable to control group (Supplementary Fig. $3 a-c)$. The levels of TG, low-density lipoprotein (LDL-c), and high-density lipoprotein (HDL-c), free fatty acid (FFA) also showed no differences (Supplementary Fig. 3d). The glucose level, glucose and insulin tolerance tests showed no significant differences (Supplementary Fig. $4 a-c$ ). To further elucidate the role of hepatic miR-126 in glucose and TG regulation, we fed control and miR-126 LKO mice with high fat diet (HFD). Hematoxylin and eosin (H\&E) staining showed that the lipid droplets accumulated in both control and miR-126 LKO mice livers after HFD fed (Supplementary Fig. 5a). The glucose tolerance and insulin sensitivity in miR-126 LKO mice were comparable to control HFD group (Supplementary Fig. 5b, c). Plasma AST, ALT, ALB, TP, and TBA levels showed no significant differences between control and miR-126 LKO mice after HFD fed (Supplementary Fig. 6a, c). There were also no differences in the levels of TG, HDL-C, LDL-C, FFA between control and miR-126 LKO mice after HFD fed (Supplementary Fig. 6d). These results indicated that hepatic miR-126 deficiency had no obvious effect on glucose and TG levels in plasma.

To elucidate role of miR-126 during liver regeneration, we examined the expression of miR-126 in the perfused hepatocytes following sham or partial hepatectomy $(\mathrm{PH})$ at $0,24,36,48$, and $72 \mathrm{~h}$ in C57BL/6 mice. The miR-126 expression peaked at $36 \mathrm{~h}$ after $\mathrm{PH}$ (Fig. 1a). Next, we performed two-thirds partial hepatectomy in control and miR-126 LKO mice. The liver to body weight significantly decreased during liver regeneration (Supplementary Fig 7a). The peak of hepatocyte proliferation in miR-126 LKO mice was significantly lower compared with control livers following $\mathrm{PH}$, as indicated by BrdU and Ki67 incorporation assay in vivo, Ki67 and EDU staining in vitro (Fig. 1b-d and Supplementary Fig 7b, c). Accordingly, the expression levels of proliferating cell nuclear antigen (PCNA) were significantly decreased at 36 and $48 \mathrm{~h}$ after $\mathrm{PH}$ compared with the corresponding control mice (Fig. 1e-g).

\author{
; https://doi.org/10.1038/s41392-020-00395-1
}

Taken together, the data indicated that deletion of miR-126 hindered hepatocytes proliferation and miR-126 was essential for liver regeneration.

MiR-126 regulates HSCs cycle and maintains HSCs quiescence through PI3K-AKT signaling pathway. ${ }^{1}$ We observed dramatic expression of p-AKT (473) and p27 in the livers of miR-126 LKO and control mice after $\mathrm{PH}$. However, the difference between two groups was not significant (Supplementary Fig. 8a-c). Forced miR-126 expression in mouse and human progenitors $B$ cells reduced p53 transcriptional activity. ${ }^{2}$ Following $\mathrm{PH}$, the levels of phospho-p53 and p21 were significantly increased at $36 \mathrm{~h}, 48 \mathrm{~h}$ after PH in miR-126 LKO livers (Fig. 1e-g; Supplementary Fig. 8d, e). These findings indicated that p53-p21 pathway might be responsible for the decreased liver regeneration in miR-126 LKO mice. After p53 inhibitor pifithrin-a (PTF-a) treatment, the expression of p-p53 and p21 were comparable between two groups after PH (Supplementary Fig. 9a, b). PTF-a treatment almost abolished the difference in PCNA expression between two groups at $48,72 \mathrm{~h}$ post-PH (Fig. 1h). PTF-a treatment also consistently eliminated the differences in $\mathrm{BrdU}$ and $\mathrm{Ki} 67$ staining between two groups at $48 \mathrm{~h}$ and $72 \mathrm{~h}$ post-PH (Fig. 1i, j). The effect of PTF-a induced p53 inhibition on liver regeneration after PH was controversial in different reported model. ${ }^{4,5}$ However, the activity of p53 in miR-126 LKO mice was significantly higher than control group. The upregulated activation of p53 pathway induced by miR-126 deficiency was contributed to the inhibition of liver regeneration. Over-expression of miR-126 in the hematopoietic system directly downregulates Cdkn2aip, ${ }^{2}$ which is a positive regulator of p53 activity. In line with this, both the mRNA and protein levels of Cdkn2aip mice were significantly increased in miR-126 LKO mice compared to control mice at 36 and $48 \mathrm{~h}$ after $\mathrm{PH}$ (Fig. 1k, I). The activity of p53-p21 pathway was also significantly downregulated after the knockdown of Cdkn2aip in isolated primary hepatocytes (Supplementary Fig. 9c). Taken together, these results validated during liver regeneration, miR126 regulated p53 signaling by targeting Cdkn2aip (Supplementary Fig. 9d).

In summary, we firstly used the hepatic conditional knockout mice to elucidate the role of miR-126 in liver and liver regeneration. Our study showed specific deletion of miR-126 in the liver decreased the rate of liver regeneration after $\mathrm{PH}$. Deficient liver regeneration in miR-126 LKO mice was a result of the activation of p53 pathway. Furthermore, deletion of miR-126 increased the expression of Cdkn2aip, which is a positive regulator of p53 activity. Disruption of p53 pathway rescued liver regeneration in miR-126-deficient mice. Therefore, miR-126 could be beneficial to liver regeneration in mice through modulating the activity of p53. The present study elucidated the role of miR-126 in liver and liver regeneration by genetic depletion, had a therapeutic potential for acute liver failure, cirrhosis, or small-forsize liver transplantations in human. 

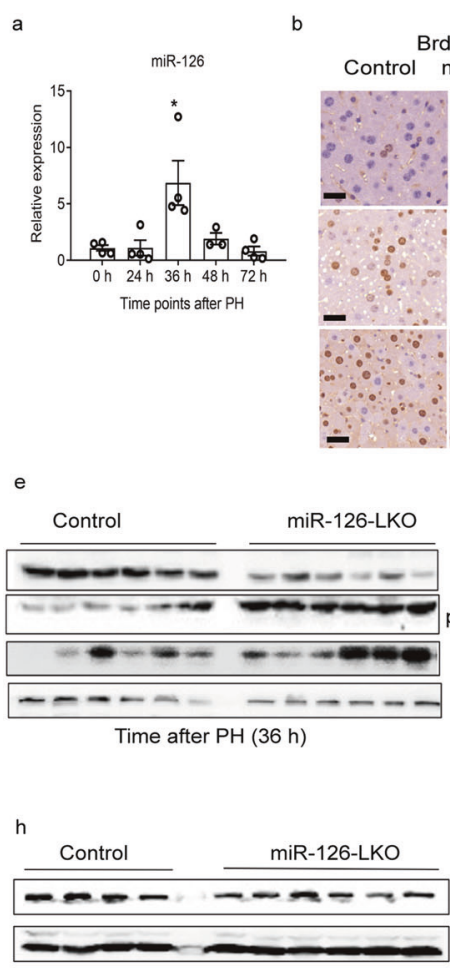

Time after PH (48 h) PFTa treatment

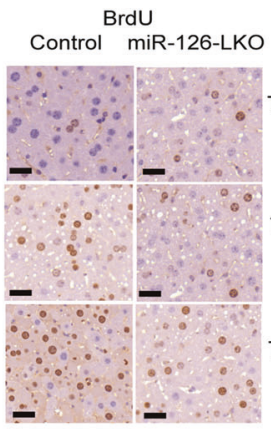

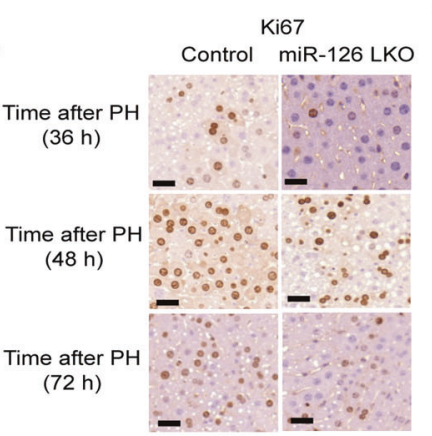

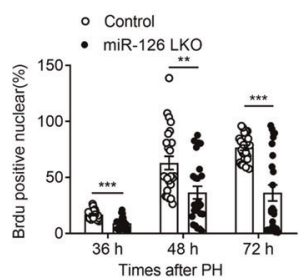

d

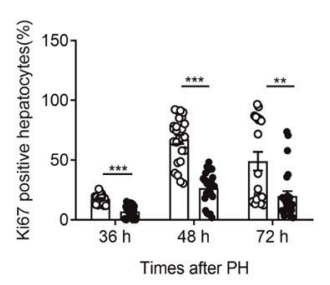

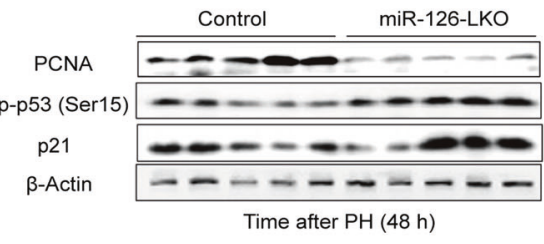
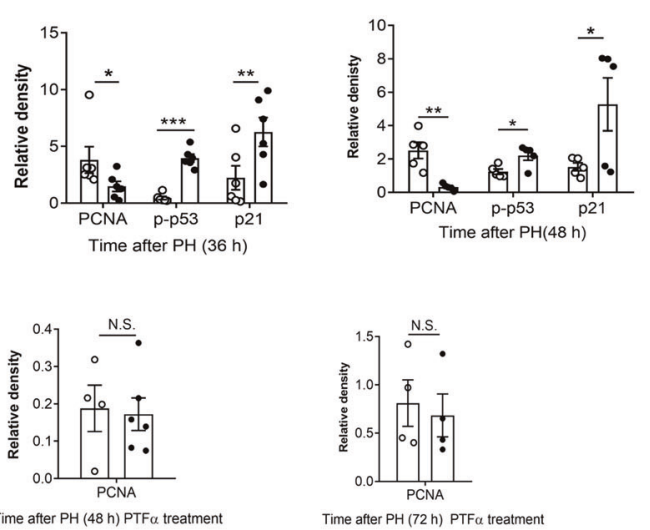

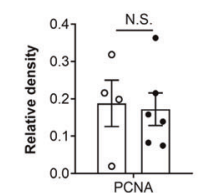

Time after PH (48 h) PTF $\alpha$ treatment
Time after PH $(72 \mathrm{~h})$ PTF $\alpha$ treatment i
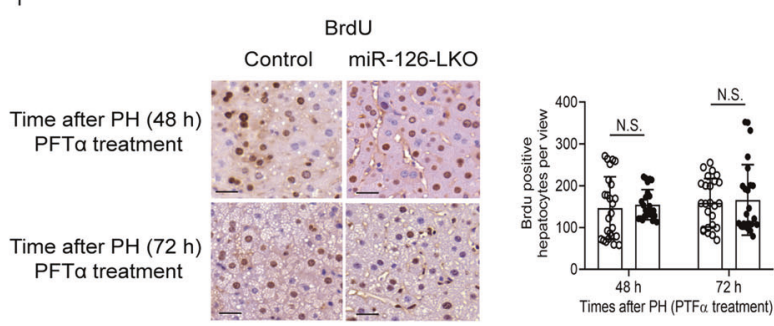

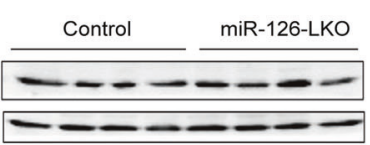

Time after PH (72 h) PFTa treatment
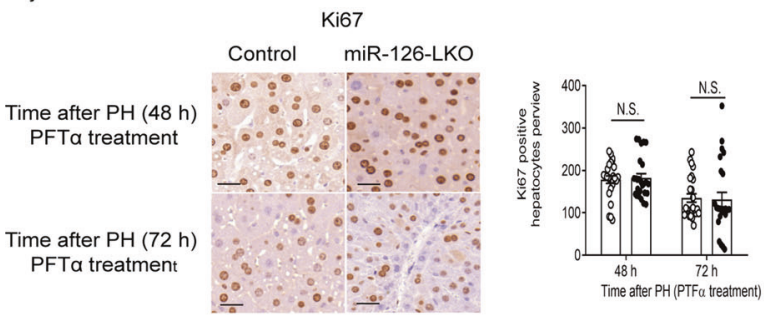

$k$

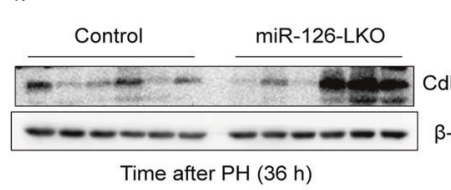

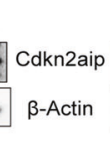

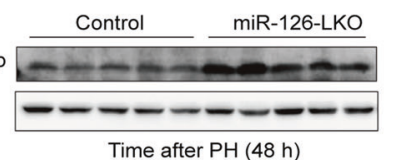

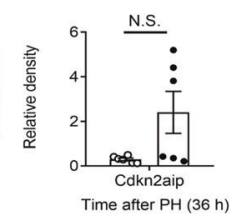
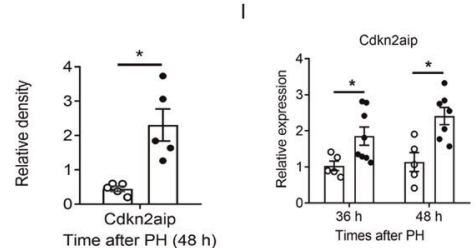

Fig. 1 MiR-126 is essential for liver regeneration through inhibiting the Cdkn2aip-p53 pathway. a Relative expression of miR-126 in the perfusd hepatoctes before and after PH at different time points. Quantifications were normalized to U6. $(0,24 \mathrm{~h}, 36 \mathrm{~h}, 48 \mathrm{~h}$, and $72 \mathrm{~h})$. b Representative IHC staining of BrdU and Ki67 positive cells in Control and miR-126 LKO livers at 36h, 48h, and 72h following PH. Scale bar: $50 \mu \mathrm{m}$. c, d The quantification figures of the IHC staining of BrdU and Ki67 in control and miR-126 LKO livers at different time points ( $36 \mathrm{~h}, 48 \mathrm{~h}$, and $72 \mathrm{~h}$ ) after PH. Five sections per mouse were examined, five mice per group. e Representative western blotting results of PCNA, p-p53, p21 in livers at different time points $(36 \mathrm{~h}, 48 \mathrm{~h})$ after PH. f, $\mathbf{g}$ The quantification figure of the western blotting analysis of PCNA, p-p53, p21 expression of livers in control and miR-126 LKO group at different time points ( $36 \mathrm{~h}, 48 \mathrm{~h}$ ) after PH. Quantifications were normalized to $\beta$-actin ( $n \geq 5$ per group). $\mathbf{h}$ Representative and quantification figures of western blotting results of PCNA expression in PFT- $\alpha$ treated control and miR126 LKO livers at $48 \mathrm{~h}, 72 \mathrm{~h}$ following PH. Quantifications were normalized to $\beta$-actin ( $n \geq 4$ per group). $\mathbf{i}$, $\mathbf{j}$ Representative quantification figures of IHC staining of BrdU and Ki67 positive cells in PFT- $\alpha$ treated control and miR-126 LKO livers at $48 \mathrm{~h}$ and $72 \mathrm{~h}$ following PH. Scale bar: $50 \mu \mathrm{m}$. Five sections per mouse were examined, five mice per group. k Representative and quantification figures of western blotting results of Cdkn2aip in livers at different time points (36h, 48h) after PH. Quantifications were normalized to $\beta$-actin ( $n \geq 5$ per group). I RT-PCR analysis of cdkn2aip expression in control and miR-126 LKO mice 36h after PH. Quantifications were normalized to $\beta$-actin ( $n \geq 5$ ). Data are presented as mean \pm SEM. ${ }^{*} P<0.05$; ${ }^{* *} P<0.01$; ${ }^{* *} P<0.001$, Wilcoxon test in $(\mathbf{k})$, student's test in $(\mathbf{a}, \mathbf{c}, \mathbf{d}, \mathbf{f}, \mathbf{g}, \mathbf{h}, \mathbf{j}$, I). PH partial hepatectomy, h hour, PCNAproliferating cell nuclear antigen, LKO liver specific knockout, N.S. no significant difference

\section{ACKNOWLEDGEMENTS}

This work is supported by National Natural Science Foundation of China (91749203, 81525010, 91949125, 81801372, 82030039), Program for Guangdong Introducing Innovative and Enterpreneurial Teams (2017ZT07S347), Innovative Team Program of
Guangzhou Regenerative Medicine and Health Guangdong Laboratory (2018GZR110103002), Natural Science Foundation of Zhejiang Province (LGD19H030001), and Key Laboratory of Aging and Cancer Biology of Zhejiang province, School of medicine, Hangzhou Normal University. 


\section{AUTHOR CONTRIBUTIONS}

L.Z., Q.J., L.L., and Z.J. designed the experiments. L.Z, F.Y., and Y.Q. conducted the experiments with the assistance of Y.Q., Y.Y., Y.W., and H.M., such as western blotting, IHC staining experiments, mouse genotyping, Q-PCR experiments, and serum biochemical tests. L.Z., L.L., and Z.J. wrote and revised the paper.

\section{ADDITIONAL INFORMATION}

The online version of this article (https://doi.org/10.1038/s41392-020-00395-1) contains supplementary material, which is available to authorized users.

Competing interests: The authors declare no competing interests.

Lingling Zhang ${ }^{1}$, Yugang Qiư ${ }^{2}$, Fan Yang ${ }^{3}$, Jiyuan Yao ${ }^{1}$, Ying Wang ${ }^{1}$, Yang Qin ${ }^{1}$, Hanchuan Mou $^{4}$, Qing Jing ${ }^{5}$, Leiming $\mathrm{Liu}^{4}$ and Zhenyu Ju'

${ }^{1}$ Key Laboratory of Aging and Cancer Biology of Zhejiang Province, Institute of Aging Research, School of Medicine, Hangzhou Normal

University, Hangzhou, China; ${ }^{2}$ School of Rehabilitation Medicine, Weifang Medical University, Weifang, China; ${ }^{3}$ School of Public Health and Management, Weifang Medical University, Weifang, China; ${ }^{4}$ Key

Laboratory of Regenerative Medicine of Ministry of Education, Regenerative Medicine and Health Guangdong Laboratory, Institute of Aging and Regenerative Medicine, Jinan University, Guangzhou, China and ${ }^{5}$ CAS Key Lab of Tissue Microenvironment and Tumor, Shanghai Institute of Nutrition and Health, Chinese Academy of Sciences, Shanghai, China

These authors contributed equally: Lingling Zhang, Yugang Qiu, Fan Yang

Correspondence: Qing Jing (jing@sibs.ac.cn) or Leiming Liu (liuleiming77@163.com) or Zhenyu Ju (zhenyuju@163.com)

\section{REFERENCES}

1. Lechman, E. R. et al. miR-126 regulates distinct self-renewal outcomes in normal and malignant hematopoietic stem cells. Cancer Cell 29, 214-228 (2016).

2. Nucera, S. et al. miRNA-126 Orchestrates an oncogenic program in B cell precursor acute lymphoblastic leukemia. Cancer Cell 29, 905-921 (2016).

3. Lin, M. W. et al. Synthetic switch-based baculovirus for transgene expression control and selective killing of hepatocellular carcinoma cells. Nucleic Acids Res. 46, e93 (2018).

4. Inoue, Y. et al. p53 May positively regulate hepatocyte proliferation in rats. Hepatology 36, 336-344 (2002).

5. Eipel, C. et al. Pifithrin-alpha induced p53 inhibition does not affect liver regeneration after partial hepatectomy in mice. J. Hepatol. 43, 829-835 (2005).

\begin{abstract}
(i) Open Access This article is licensed under a Creative Commons Attribution 4.0 International License, which permits use, sharing, adaptation, distribution and reproduction in any medium or format, as long as you give appropriate credit to the original author(s) and the source, provide a link to the Creative Commons license, and indicate if changes were made. The images or other third party material in this article are included in the article's Creative Commons license, unless indicated otherwise in a credit line to the material. If material is not included in the article's Creative Commons license and your intended use is not permitted by statutory regulation or exceeds the permitted use, you will need to obtain permission directly from the copyright holder. To view a copy of this license, visit http://creativecommons. org/licenses/by/4.0/.
\end{abstract}

(c) The Author(s) 2021 